\title{
Isolated Handwritten Roman Numerals Recognition Using Methods Based on Radon, Hough Transforms and Gabor Filter
}

\author{
R. Salouan, S. Safi and B. Bouikhalene \\ Department of Mathematic and Informatic Polydisciplinary Faculty \\ Sultan Moulay Slimane University \\ Beni Mellal, MOROCCO
}

\begin{abstract}
This paper presents for isolated handwritten Roman numerals recognition a research interested for carrying out both comparisons between the performances in terms of precision and rapidity, the first comparison is realized between four hybrid methods used to extract the features from numerals that are the zoning combined with Radon transform in first time, then combined with Hough transform in second time, then combined with Gabor filter in third time, finally combined in the same time with all these three descriptors, while the other comparison is performed between three classifiers which the first one is neuronal that is the multi-layer perceptron whereas the second is probabilistic that is hidden Markov model, the third classifier is realized via a combination between these both classifiers. For this purpose we have employed for pre-processing each numeral image the median filter, the thresholding, the centering and the edge detection techniques.
\end{abstract}

Keywords: Isolated handwritten Roman numerals, median filter, thresholding, centering, edge detection, zoning method, Radon transform, Hough transform, Gabor filter, multi-layer perceptron, hidden Markov model

\section{Introduction}

In recent years the Optical Character Recognition (OCR) became a very dynamic domain in terms of innovation and creativity in wide sense of word due to the increasing use of computers in various fields such as postal code recognition, automatic text entry into computer, language processing, automatic sorting of postal mail, bank cheques recognition, etc.

In practice, there is two principal categories in each OCR which the first one is intersted to printed characters while the second is focused to those handwritten, in this context, it is obviously that the last category is more difficult compared to the first taking into account that the intraclass variance is high in reason of the different forms associated to a same character, because there is a particular writing style of each person, furthermore a certain character can be written in many different forms even by a same given person. For this reason, it is necessary to use some efficient techniques in each one of the three principal phases forming a certain recognition system which are the pre-processing used to improve the quality of the character image, and features extraction exploited to convert the image to a vector contains numerical values that not are only of the information characterizing the character. Finally there is the recognition phase employed in order to train all the character images of the learning base and classify those of the test database.

Moreover, we have centered our attention in this study on isolated handwritten Roman numerals recognition, in this sense, for achieving with success this task, we have opted in the first phase the median filter, the thresholding, the centering and the edge detection techniques, concerning the second phase we have exploited four hybrid methods based on a combination between zoning technique and Radon transform firstly then between 
zoning with Hough transform secondly then between zoning with Gabor filter, afterwards all these four descriptors are in the same time combined between them, finally in order to recognize each numeral image we have employed three classifiers which are the multilayer perceptron and hidden Markov model then a hybridization between these both classifiers. In fact, more precisely the main contribution in this work is to compare between the performances of these four hybrid methods of features extraction in one side and between these three classifiers on the other side, in order ultimately to deduct the most performing recognition system in terms of precision and rapidity.

Besides that, there are many studies have been proposed in the literature for recognizing Indian, Latin or Arabic numerals and characters by using the zoning method [1-3] the Radon [4-6] and Hough [7-8] transforms, the Gabor filter [9-10] the multi-layer perceptron [11-15] the hidden Markov model [16-19].

Hence, the remainder of the organization of this paper is as follows. In Section 2, the methodology that we have opted in this study is given. In Section 3, the techniques preprocessing are presented. In Section 4, features extraction methods are described. A Section 5 deal describes the numerals recognition procedures. Experimental methods and results are given in Section 6. Lastly, the conclusion and scope of further work are described in Section 7.

\section{Methodology}

The recognition systems which are we have used can be presented as follow:

Image numeral
Pre-processing
(Median filter-Thresholding-Centering-Edge detection)
Features extraction
(Zoning method - Radon transform - Hough transform - Gabor filter)
Recognition
(Multi-layer perceptron - Hidden Markov model)
Output numeral

Figure 1. The Proposed Recognition Systems

\section{Pre-processing}

Pre-processing is undoubtedly an important part of each optical character recognition system especially when it comes of handwritten characters, its objective is to render the numeral image in a best quality in terms of removal of noise and of redundant and needless information in character image, for this goal we have employed the median filter used for filtering each numeral image, the thresholding which serves to render the numeral image contains only the black and white colours according a preset threshold, the centering exploited to put the numeral justly in center of image and the edge detection performed to find the edge of numeral. 

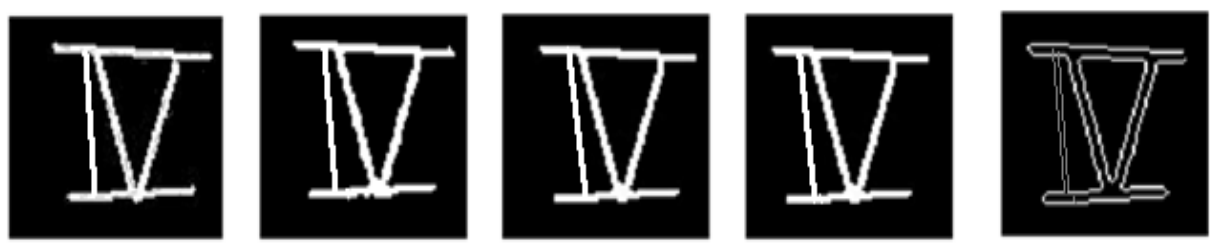

Figure 2. Different Techniques used for Pre-Processing of Handwritten Roman Numeral IV

\section{Feature Extraction}

Features extraction is really considered as a one of the major problems of character recognition. In fact the performance of character recognition system is depends enormously on proper features extraction, in fact, a certain method is very effecient if it achieves a great discrimination interclasses and a small discrimination intraclasses of the fact that all of that effectively allows to render the recognition phase will be easier. For this finality, in order to realize all that with success, we have used four hybrid methods which are respectively:

- Zoning method combined with Radon transform.

- Zoning method combined with Hough transform.

- Zoning method combined with Gabor filter.

- Zoning method combined with Radon, Hough transforms and Gabor filter.

\subsection{The Zoning Method}

Firstly, given a black image that contains a numeral written in white, the zoning method consists to divide this image to a several zones then calculating in each one of them the number of white pixels, all these numbers are stocked in a vector, that is to say image is converted to a vector has a number of components equal to that of zones.

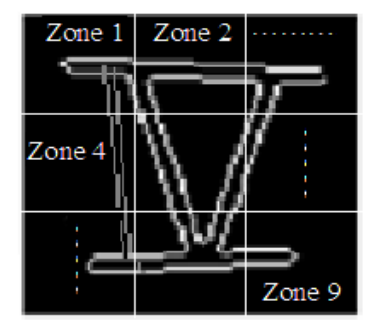

Figure 3. Zoning Method of Handwritten Roman Numeral IV

\subsection{Radon Transform}

Due to its ability to represent efficiently significant features of an image Radon transform have found wide applications in the fields of image processing and pattern recognition [20-21]. The Radon transform computes the projections of an image $f(x, y)$ along some specified and pre-determined radial directions according a specific set of angles. The Radon transform is defined as follow:

$$
T_{R_{f}}(\rho, \theta)=\iint f(x, y) \delta(x \cos \theta+y \sin \theta-\rho) d x d y
$$

Where is the impulse of Dirac given by: 


$$
\delta(x)=\left\{\begin{array}{lll}
1 & \text { if } & x=0 \\
0 & & \text { elsewhere }
\end{array}\right.
$$

Furthermore, the Radon transform needs as input parameters a number of directions denoted $n$ according to which the integrals are calculated generally fixed to $2 \pi$ and a pitch denoted $p \quad$ which is thus automatically fixed to $\frac{2 \pi}{n}$ in order to ensure a homogeneous distribution in $\theta$.

\subsection{Hough Transform}

The Hough Transform (HT) [22] is a technique commonly used in image processing. It is known for its capacity to detect in a given image among other things, straight lines in digital images. In polar coordinates, a straight line can be described via the equation:

In the HT space, a line is represented in its normal form by:

$$
\rho=x \cos \theta+y \sin \theta \quad 0 \leq \theta \leq 2 \pi
$$

The above equation describes a mapping of a point in the Cartesian coordinate plane $(x, y)$ to the sinusoidal curve in the polar coordinats $\rho-\theta$ plane

Each feature point $(\mathrm{xi}, \mathrm{yi})$ votes then for a sinusoid of points in the parameter space (accumulator). Where these sinusoids cross, there are higher accumulator values. Localizing maxima in the accumulator is equivalent to finding the existing lines.

The line detection in a binary image using the Hough transform algorithm can be summarized as follows:

1) Define the Hough transform $\rho_{\text {min }}, \rho_{\max }, \theta_{\min }$ and $\theta_{\max }$

2) Quantify the plane $\quad \rho-\theta$ into cells by forming an accumulator cells array $A(\rho, \theta)$

where $\rho_{\text {is between }} \rho_{\min }$ and $\rho_{\max }$ and $\theta$ is between $\theta_{\min }$ and $\theta_{\max }$

3) Initialize each element of an accumulator cells array $A$ to zero.

4) For each pixel of numeral in a binary image, perform the following:

For each value of $\theta$ from $\theta_{\min }$ to $\theta_{\mathrm{max}}$, calculate the corresponding $\rho$ using equation:

$$
\rho=x \cos \theta+y \sin \theta
$$

Round off the $\rho$ value to the nearest interval value.

Increment the accumulator array element $A(\rho, \theta)$

Detect best line candidates as local maxima in the accumulator cell array.

Hence, The following example presents graphically the Radon and Hough transforms for each isolated handwritten Roman numeral knowing that $\theta=\left\{0, \frac{\pi}{18}, \frac{\pi}{36} \ldots . \pi\right\}$ 


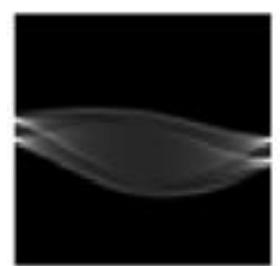

I(Radon)

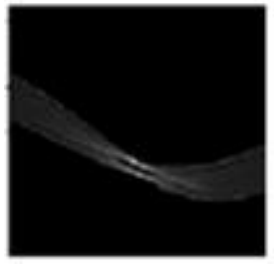

I (Hough)
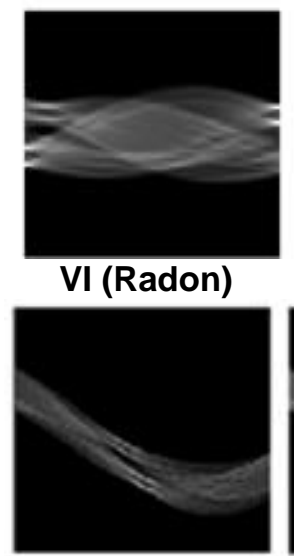

VI (Hough)

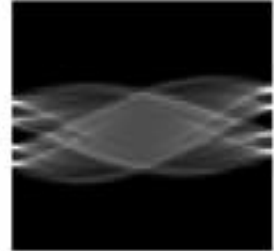

II (Radon)

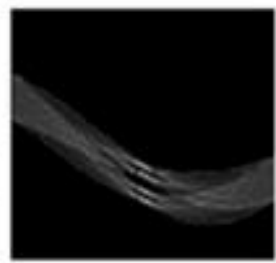

II (Hough)
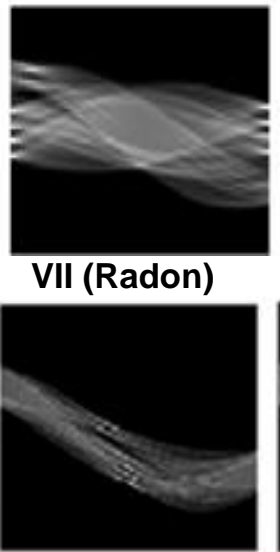

VII (Hough)

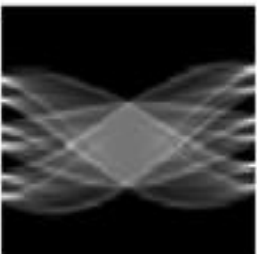

III(Radon)

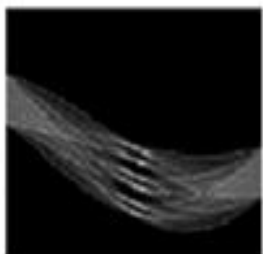

III(Hough)
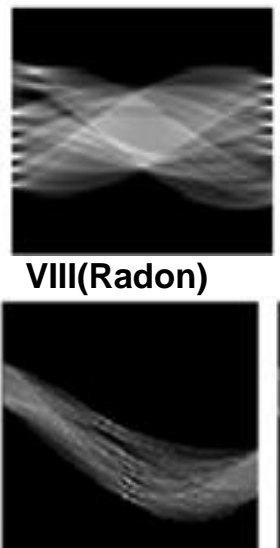

VIII(Hough)
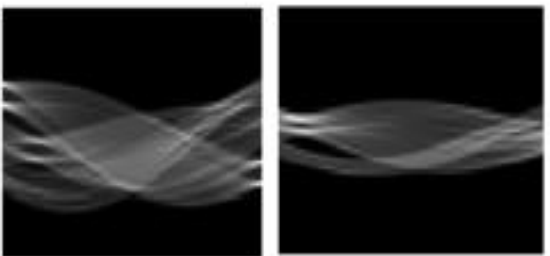

IV (Radon)

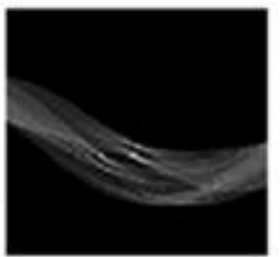

IV (Hough)
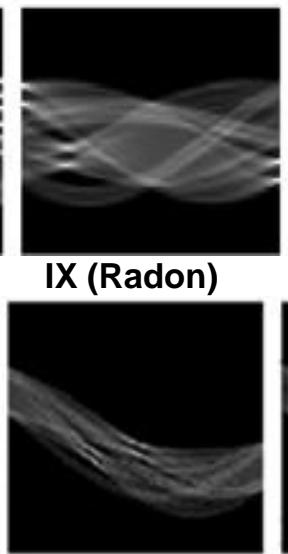

IX (Hough)
V (Radon)

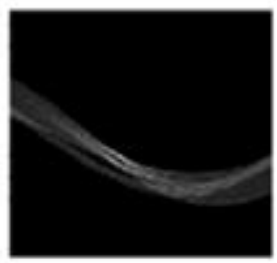

V (Hough)

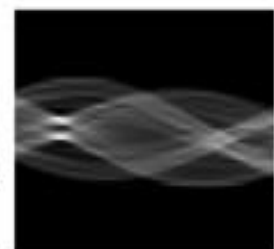

X (Radon)

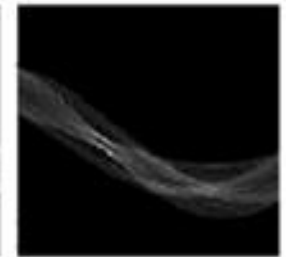

X(Hough)

Figure 4. Accumulators of Radon and Of Hough Transforms For Each Handwritten Roman Numeral

\subsection{Gabor Filter}

Gabor filters is used in many various fields depending on pre-processing image such as image compression, edge detection, texture analysis, object recognition and facial recognition.

Gabor filter is a Gaussian kernel function modulated by a sinusoidal plane wave [23-24] and is defined as follows:

$$
G(x, y, \theta, f)=e^{\left(\left(\frac{-1}{2}\left(\frac{x^{\prime}}{o_{x^{\prime}}}\right)^{2}+\left(\frac{y^{\prime}}{o_{y^{\prime}}}\right)^{2}\right)\right.} \cos \left(2 \pi f x^{\prime}\right)
$$

With:

$$
x^{\prime}=x \cos \theta+y \sin \theta
$$

$y^{\prime}=-y \cos \theta-x \sin \theta$

$\sigma_{x}, \sigma_{y}$ are the variances of the $x$ and $y$ axes respectively

$f$ is the frequency of the sinisouide function.

$\theta$ is the orientation of Gabor filter.

$G$ is the output of Gabor filter for each different values of f and $\theta$ 


\section{Recognition}

\subsection{Simple Classifiers}

5.1.1 Multi-layer Perceptron : The Neural Network presented in this figure is a multilayer perceptron that we have used in this study.

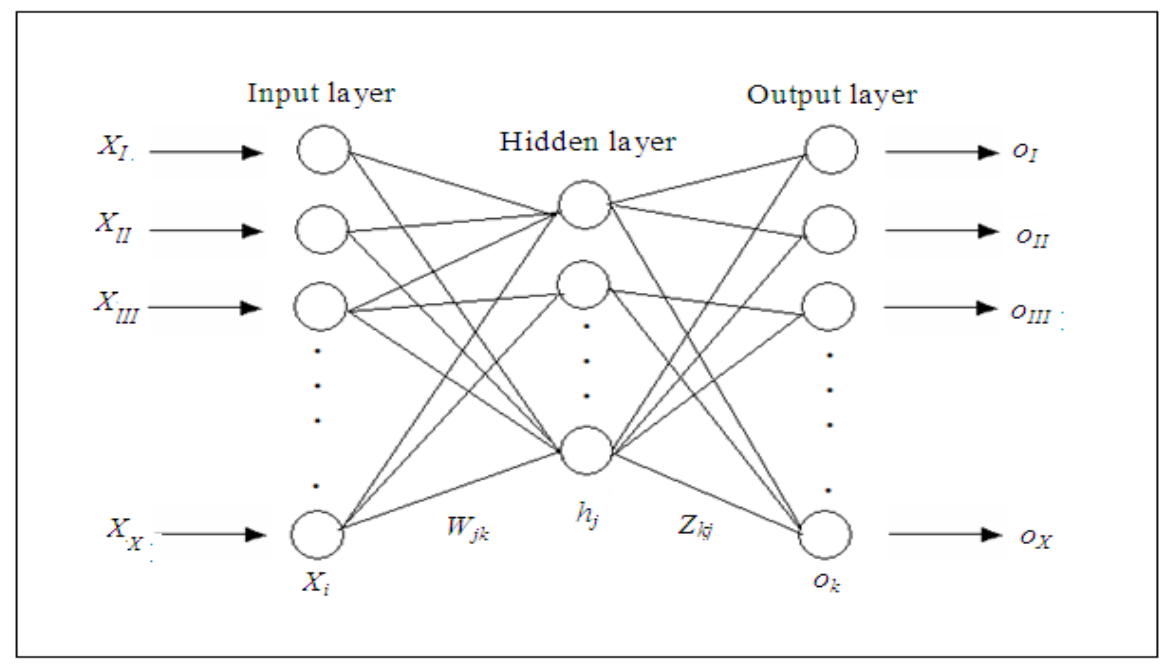

Figure 5. Architecture of Multi Layer Perceptron

The multi-layer perceptron neural networks have been applied successfully to solve some difficult and diverse problems by training them in supervised manner with a highly popular algorithm known as error back-propagation algorithm, Concerning the , the MLP that we have used contains the following elements :

- An input layer of 10 vectors who's each of them represents a handwritten Roman numeral $X_{i}$.

- A hidden layer of 7 activations neural $h_{j}$

- An output layer of 10 activations neural $\boldsymbol{O}_{k}$

$-7 \times 10$ connections between input layer and hidden layer, each weighted by

$-10 \times 7$ connections between hidden layer and output layers, each weighted by

The learning of multi-layer perceptron is achieved by the back propagation algorithm in five principal phases which

Phase 1. Initialize the network, with all wight $W$ and $Z$ set to random numbers between -1 and +1 .

Phase 2. Present firstly the vectors $X_{i}$ whose all numerals are converted and obtain the real outputs which are calculated by a sigmoid function :

$$
f(x)=\frac{1}{1+e^{-X_{i}}} .
$$

Phase 3. Compare between each real output and each desired output which is a10element vector with a 1 in the position of the numeral it represents, and 0's everywhere else, for example, the numeral "II" is to be represented by a 1 in the second element (because "II" is the second numeral of the Romain numerals), and 0's elsewhere.

Phase 4. Propagate the error backwards: 
i) Correct the weight of output layer

ii) Correct the input weight

Phase 5. Take the average difference between the desired and the output vector to calculate the error.

Phase 6. Repeat from phase 2 until the real output converges to the desired output.

After the learning of MLP, for classifying an test numeral, that is presented as a vector of test $X_{\text {test }}$ the value of $f\left(X_{\text {test }} W^{*}\right)=h_{j}$ is calculated then the Euclidean distance between the value of $f\left(h_{j} Z^{*}\right)=o_{k}$ ( $f$ is the sigmoid function) and each ${ }^{o_{k}}$ for $j=\{I, I I \ldots . X\}$ is calculated.

Therefore, the recognition will be assigned finally to the numeral that is very nearest to test numeral.

5.1.2 The Hidden Markov Model: Hidden Markov model [25] is a probabilistic tool which considered as a one of the most effecient methods in pattern recognition. In fact, it offer many important properties for modeling characters or words. Among these properties is the existence of efficient algorithms allowing to learn automatically the models without any need of labeling of data's. The HMM is based on a doubly stochastic processes whose the first one is hidden while the second is observable. The transition of the process from an actual state to a next is based on this underlying process. The observable outputs or the observations are generated by other stochastic process which is given by probabilities.

The HMM with a discrete observation symbol is defined by $\lambda=(A, B, \pi)$ where $A$ is the matrix of the probabilities of transitions, $B$ is the matrix of the probabilities of observations, and $\pi$ is the vector probability of initial states.

$N$ : The number of states $s_{1}, S_{2} \ldots \ldots s_{N}$

$T:$ The number of observations.

$q_{t}$ The state of the process at the time $t$

$q_{t}=\left\{s_{1}, s_{2}, \ldots ., s_{N}\right\}$

$o_{t}$ The observation at the time $t \quad o_{t}=\left\{v_{1}, v_{2}, \ldots ., v_{M}\right\}$

$M$ The size of observations $v_{1}, v_{2} \ldots . . v_{M}$

$$
\begin{aligned}
& A=a_{i j}=P\left(s_{j} / s_{i}\right) \quad, \quad \sum_{j=1}^{N} a_{i j}=1 \\
& \pi=\pi_{i}=P\left(s_{i}\right) \quad, \quad \sum_{j=1}^{N} \pi_{i}=1 \\
& B=b_{j}(k)=P\left(o_{t}=v_{k} / q_{t}=s_{i}\right) \\
& \sum_{k=1}^{M} b_{j}(k)=1
\end{aligned}
$$




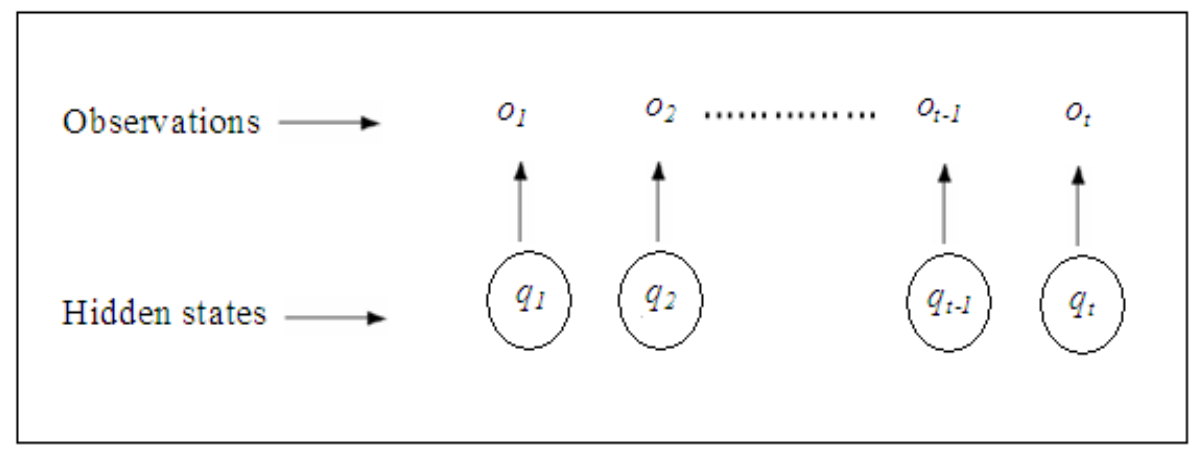

Figure 6. Example of Hidden Markov Model

The hidden Markov model with a continuous observation is defined by $\lambda=(\mathrm{A}, \mathrm{B}, \sigma \mathrm{i}, \mu \mathrm{i})$ where $\mu \mathrm{i}$ and $\sigma \mathrm{i}$ are respectively the mean and the standard deviation of the state $i$ of the Gaussian function that used for to generate the probability of observation:

$$
b_{j}(k)=\operatorname{Pr} o b\left(o_{t}=v_{k} / o_{t}=s_{j}\right)=\frac{1}{\sigma_{i} \sqrt{2 \pi}} e^{\frac{\left(o_{t}-\mu_{i}\right)^{2}}{2 \sigma_{i}^{2}}}
$$

In the context of the learning - classification phase, it carried as follow:

In the learning phase, each numeral image that is converted to a vector in the features extraction phase is used as an observation vector of an initial own HMM of this numeral in order to determine the probability that generated this observation. Then this model is trained for maximizing this probability by using the Baum-Welch algorithm. All these trained models (optimal models) of all numerals are saved for to form a learning base. In the classification phase, an unknown numeral (test numeral) is presented as a vector of observation. Then the probability generated by this observation is calculated by all the optimal models by the forward algorithm. The recognition will be given to the numeral with highest probability.

\subsection{Hybrid System MLP+HMM}

This hybridization consists to consider each output vector associated to a certain numeral which is produced by the MLP as a vector of observation of a HMM $\lambda_{j}=\left(A_{j}, B_{j}, \sigma_{i, j}, \mu_{i, j}\right) \quad$ for $j=\{I, I I \ldots . X\}$ that is must be trained by Baum-Welch algorithm for getting an optimal model $\lambda_{j}^{*}$ and a maximum probability(likelihood) $P_{j}^{*}=P\left(O_{j} / \lambda_{j}^{*}\right)$ generating this observation, then all these optimal parameters $\left(\lambda_{j}^{*}, P_{j}^{*}\right)$ will be stocked for forming a learning database which contains also the optimal weight matrixes $W^{*}$ and $Z^{*}$ of MLP, afterwards concerning the classification phase an unknown handwritten Roman numeral $X_{\text {test }}$ is presented then the value of $f\left(X_{\text {test }} W^{*}\right)=h_{i}$ and the value of $f\left(h_{i} Z^{*}\right)=o_{t}$ is determined, then the distance $d\left(P_{j}^{*}, P_{t}\right)=P_{j}^{*}-P_{t}$ is calculated for each $j=\{I, I I \ldots . . X\}$ where $P_{t}=P\left(O_{t} / \lambda_{j}^{*}\right)$ is obtained by Forward algorithm) is calculated.

The evaluation of recognition is ultimately prrsented by :

$$
\operatorname{Calss}\left(X_{\text {test }}\right)=\underset{j=I, I \ldots \ldots X}{\arg }\left(\min \left(d\left(P_{j}^{*}-P_{t}\right)\right)\right.
$$




\section{Experiments and Results}

Firstly, we present an example of some isolated handwritten Roman numerals.

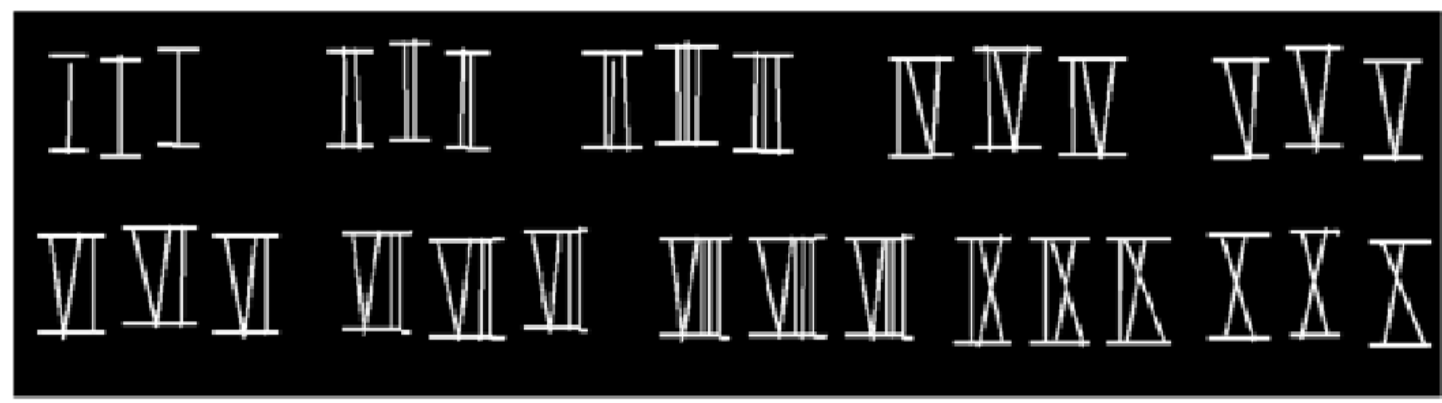

Figure 7. Example of Some Isolated Handwritten Roman Numerals

The desired aim is to compare between the performances in terms of recognition rate (precision) and recognition time (rapidity) of:

Four hybrid methods of features extraction that are:

- Zoning + Radon $(\mathrm{Z}+\mathrm{R})$.

- Zoning + Hough $(\mathrm{Z}+\mathrm{H})$.

- Zoning + Gabor $(\mathrm{Z}+\mathrm{G})$.

- Zoning + Radon + Hough + Gabor $(\mathrm{R}+\mathrm{H}+\mathrm{G}+\mathrm{Z})$.

In one hand and between the performances of three classifiers which are:

- Multi-Layer Perceptron (MLP).

- Hidden Markov Model (HMM).

- Multi-Layer Perceptron + Hidden Markov Model (MLP+HMM).

On the other hand.

Therefore in order to achieve these comparisons, we have used the following data's:

- Each numeral image has a size equal to $45 \times 45$ pixels.

- The number of all images of learning and of test that we have used is equal to 3000 images.

- The number of zones whose each image numeral is divided is equal to 9 zones.

Therefore each numeral image is finally converted to a vector by the following relationship:

$$
X=X_{\text {zoning }} \cup X_{\text {Radon-Hough-Gabor }}
$$

Therefore, we grouped the values that we obtained of the recognition rate $\tau_{n}$ of each numeral (given in $\%$ ) and of the global rate $\tau_{g}$ (given in $\%$ ) also the global recognition time $t_{g}$ (given in second), i.e., of all numerals for each hybrid method of features extraction and each classifier in the following Table :

Table 1. The Recognition Rate of Each Numeral and Global Recognition Rate and Global Time of Execution for Each Features Extraction Method and for Each Classifiers

\begin{tabular}{|l|l|l|l|l|l|l|l|l|l|l|l|l|}
\hline $\mathrm{N}$ & \multicolumn{3}{|c|}{$\tau_{n}(\mathrm{MLP})$} & \multicolumn{4}{c|}{$\tau_{n}(\mathrm{HMM})$} & \multicolumn{5}{c|}{$\tau_{n}(\mathrm{MLP}+\mathrm{HMM})$} \\
\cline { 2 - 11 } & $\mathrm{Z}+\mathrm{R}$ & $\mathrm{Z}+\mathrm{H}$ & $\mathrm{Z}+\mathrm{G}$ & $\mathrm{Z}+\mathrm{R}+\mathrm{H}+\mathrm{G}$ & $\mathrm{Z}+\mathrm{H}$ & $\mathrm{Z}+\mathrm{G}$ & $\mathrm{Z}+\mathrm{R}$ & $\mathrm{Z}+\mathrm{R}+\mathrm{H}+\mathrm{G}$ & $\mathrm{Z}+\mathrm{G}$ & $\mathrm{Z}+\mathrm{R}$ & $\mathrm{Z}+\mathrm{H}$ & $\mathrm{Z}+\mathrm{R}+\mathrm{H}+\mathrm{G}$ \\
\hline
\end{tabular}




\begin{tabular}{|c|c|c|c|c|c|c|c|c|c|c|c|c|}
\hline & 83.55 & 82.78 & 85.64 & 94.05 & 81.65 & 80.55 & 83.15 & 93.05 & 88.15 & 87.10 & 90.18 & 98.95 \\
\hline & 81.34 & 80.12 & 83.54 & 92.34 & 81.90 & 80.65 & 82.10 & 92.05 & 86.18 & 85.25 & 87.12 & 98.08 \\
\hline & 82.67 & 81.85 & 83.60 & 93.15 & 81.45 & 80.35 & 82.23 & 92.30 & 87.34 & 86.30 & 88.15 & 98.85 \\
\hline & 82.78 & 80.14 & 83.48 & 92.97 & 80.48 & 80.45 & 81.60 & 92.18 & 85.67 & 84.18 & 87.62 & 98.34 \\
\hline & 83.12 & 81.76 & 84.55 & 93.58 & 81.13 & 80.76 & 83.45 & 92.95 & 86.85 & 85.34 & 87.50 & 98.05 \\
\hline & 81.58 & 80.67 & 82.37 & 92.85 & 80.53 & 80.55 & 82.30 & 92.05 & 86.55 & 85.15 & 87.25 & 98.15 \\
\hline & 84.78 & 83.45 & 85.34 & $\begin{array}{l}94.88 \\
\end{array}$ & 82.34 & 81.43 & 83.34 & 93.34 & 88.87 & 86.17 & 89.67 & 999.30 \\
\hline & 85.85 & 83.75 & 86.52 & 96.67 & 83.67 & 81.88 & 84.42 & 94.85 & 89.10 & 87.34 & 90.40 & 99.67 \\
\hline & 84.45 & 82.65 & 85.78 & 96.34 & 82.44 & 81.56 & 83.45 & 94.75 & 89.34 & 86.25 & 89.75 & 99.34 \\
\hline & 83.40 & 81.15 & 84.70 & 95.95 & 81.76 & 80.25 & 82.10 & 94.87 & 88.34 & 85.20 & 89.85 & 99.15 \\
\hline & 83.35 & 81.83 & 84.55 & 94.28 & 81.73 & 80.84 & 82.81 & 93.24 & 87.64 & 85.82 & 88.75 & 98.80 \\
\hline$i_{g}$ & 68.62 & 73.40 & 80.25 & 97.51 & 41.83 & 43.14 & 48.42 & 68.17 & 110.64 & 114.87 & 119.74 & 145.61 \\
\hline
\end{tabular}

The associated graphical representation to recognition rate $\tau_{n}$ is:

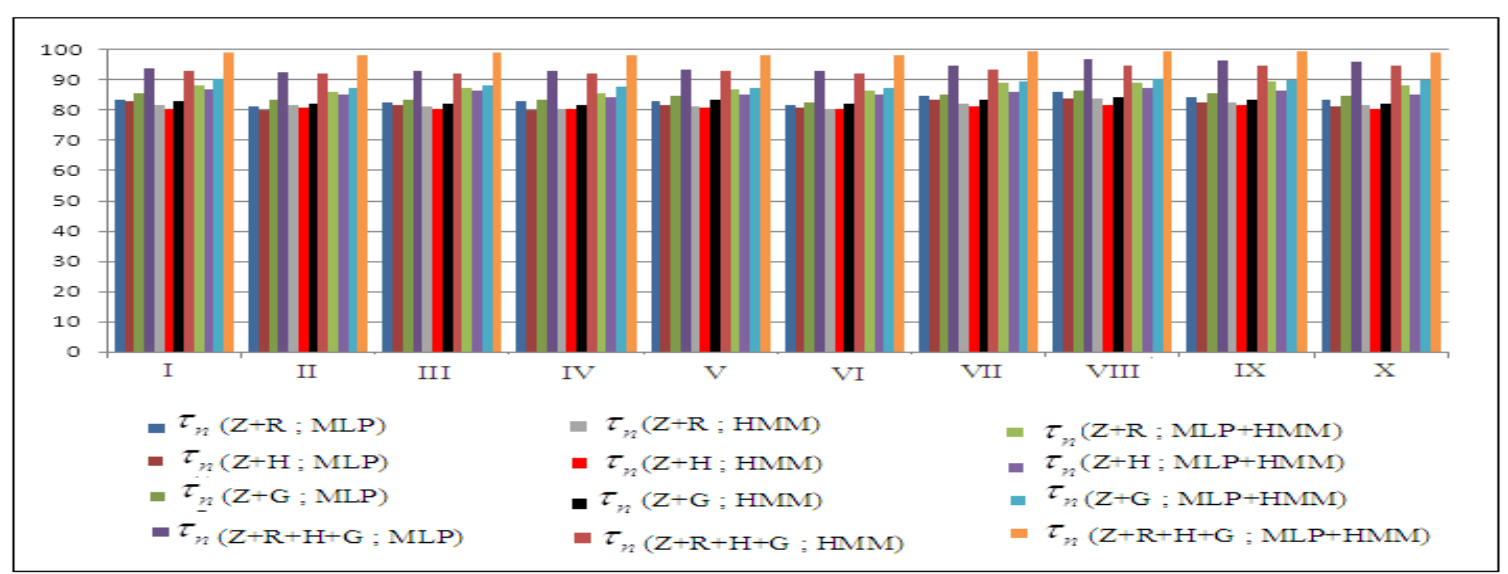

Figure 8. The Recognition Rate of Each Numeral for Each Features Extraction Method and for Each Classifier

\section{Analysis and comments:}

Considering all the results that we have obtained, we can to conclure that :

- The numerals the most correctly recognized are : I, II, VIII, IX.

- The numerals the less correctly recognized are :IV, $X$.

- The most precise hybrid method of features extraction is zoning combined in the same time with Radon, Hough transforms and Gabor filter followed by zoning combined with Gabor filter then zoning combined with Radon transform then zoning combined with Hough transform.

- The most precise classifier is the hybrid system multi-layer perceptron combined with hidden Markov model followed by multi-layer perceptron then hidden Markov model.

The associated graphical representation to recognition rate $\tau_{n}$ is: 


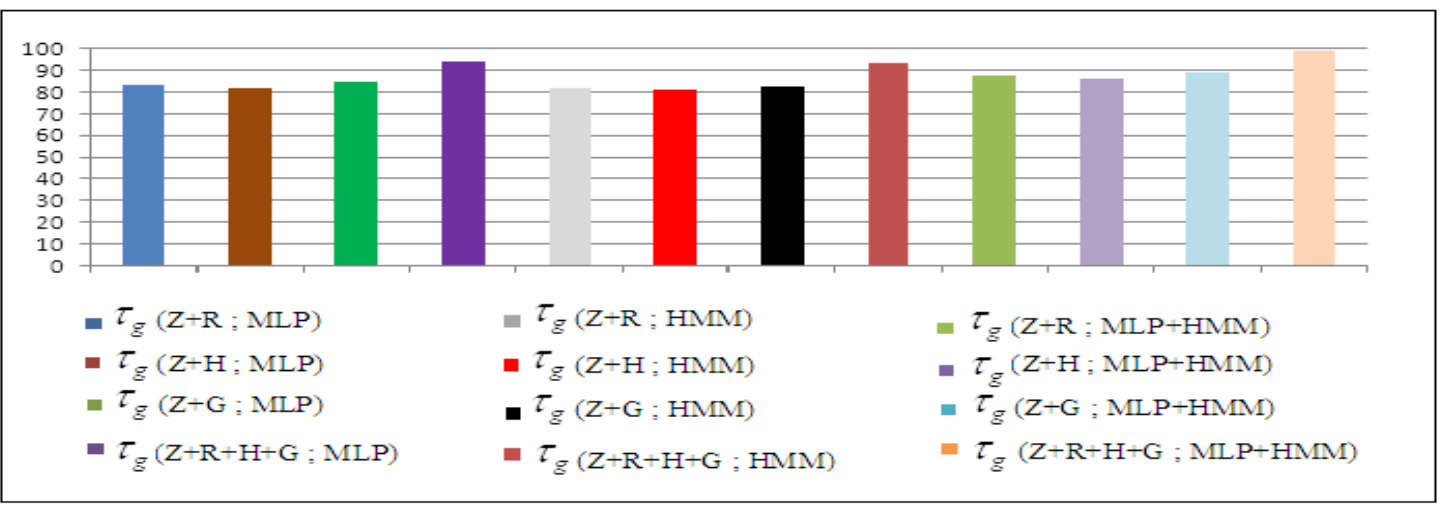

Figure 9: The Global Recognition Rate for Each Features Extraction Method and for Each Classifier

\section{$\diamond$ Analysis and comments:}

Taking into account all the results that we obtained, we concluded that the most precise recognition system is that which contains a zoning method combined with Radon, Hough transforms and Gabor filter as a features extraction and the multi-layer perceptron combined with hidden Markov model as a classifier. Moreover the associated graphical representation to time of execution is presented as follow:

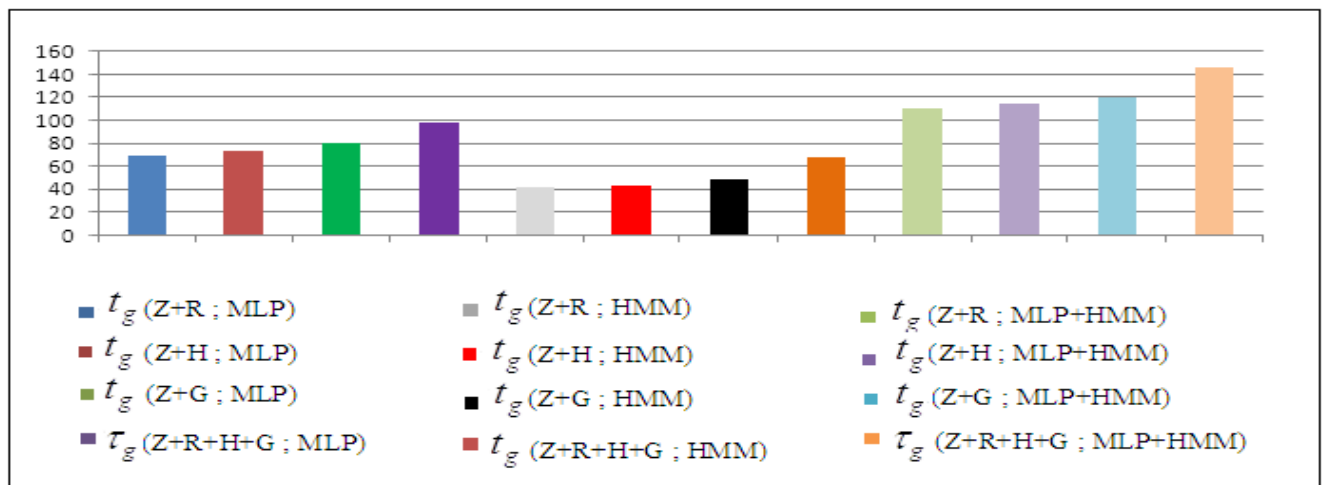

\section{Figure 10. The Global Time of Execution for Each Features Extraction} Method and Each Classifier

\section{$\diamond$ Analysis and comments:}

Having regard the results presented in figure 10, we concluded that the most fast hybrid method is the zoning combined with Radon transform then with Hough then with Gabor filter, finally zoning combined in the same time with all these descriptors in one side and the fastest classifier is hidden Markov model followed by multi-layer perceptron followed by multi-layer perceptron combined with hidden Markov model on the other side. For fixing this idea, we note the difference of precision between $h m_{1}, c l_{1}$ and $h m_{2}, c l_{2}$

$$
\Delta P=\tau_{g, h m_{1}, c l_{1}}-\tau_{g, h m_{2}, c l_{2}}
$$

- If $\Delta P>0$ we have a gain of precision. In this case $\Delta P$ is a rate of growth of precision.

- If $\Delta P<0$ we have a loss of precision. In this case $\Delta P$ is a rate of decay of precision. In a like manner, we note the difference of rapidity between $h m_{1}, c l_{1}$ and $h m_{2}, c l_{2}$ 


$$
\Delta \boldsymbol{R}=\boldsymbol{t}_{g, h m_{1}, c l_{1}}-\boldsymbol{t}_{g, h m_{2}, c l_{2}}
$$

- If $\Delta R>0$ we have an advancement in rapidity. In this case $\Delta R$ is a rate of decay of rapidity.

- If $\Delta R<0$ we have a delay in rapidity. In this case $\Delta R$ is a rate of growth of rapidity. Where $h m$ means to hybrid method and $\mathrm{cl}$ to classifier. Hence, the table below presents different values of $\Delta P$ (gain of precision) and $\Delta R$ (lateness of time of execution).

Table 2. Various Values of Difference of Precision and of Rapidity

\begin{tabular}{|c|c|c|}
\hline$h m_{1}, c l_{1} ; h m_{2}, c l_{2}$ & $\Delta P(\%)$ & $\Delta R($ second) \\
\hline $\mathrm{Z}+\mathrm{R}+\mathrm{H}+\mathrm{G}, \mathrm{MLP}+\mathrm{HMM} ; \mathrm{Z}+\mathrm{R}, \mathrm{MLP}+\mathrm{HMM}$ & 11.16 & 30.74 \\
\hline $\mathrm{Z}+\mathrm{R}+\mathrm{H}+\mathrm{G}, \mathrm{MLP}+\mathrm{HMM} ; \mathrm{Z}+\mathrm{H}, \mathrm{MLP}+\mathrm{HMM}$ & & 25.87 \\
\hline $\mathrm{Z}+\mathrm{R}+\mathrm{H}+\mathrm{G}, \mathrm{MLP}+\mathrm{HMM} ; \mathrm{Z}+\mathrm{G}, \mathrm{MLP}+\mathrm{HMM}$ & 10.05 & 48.10 \\
\hline $\mathrm{Z}+\mathrm{R}+\mathrm{H}+\mathrm{G}, \mathrm{MLP}+\mathrm{HMM} ; \mathrm{Z}+\mathrm{R}+\mathrm{H}+\mathrm{G}, \mathrm{MLP}$ & & 76.99 \\
\hline $\mathrm{Z}+\mathrm{R}+\mathrm{H}+\mathrm{G}, \mathrm{MLP}+\mathrm{HMM} ; \mathrm{Z}+\mathrm{R}, \mathrm{MLP}$ & 15.45 & 72.21 \\
\hline $\mathrm{Z}+\mathrm{R}+\mathrm{H}+\mathrm{G}, \mathrm{MLP}+\mathrm{HMM} ; \mathrm{Z}+\mathrm{H}, \mathrm{MLP}$ & 16.97 & 65.36 \\
\hline $\mathrm{Z}+\mathrm{R}+\mathrm{H}+\mathrm{G}, \mathrm{MLP}+\mathrm{HMM} ; \mathrm{Z}+\mathrm{G}, \mathrm{MLP}$ & 14.25 & 77.44 \\
\hline $\mathrm{Z}+\mathrm{R}+\mathrm{H}+\mathrm{G}, \mathrm{MLP}+\mathrm{HMM} ; \mathrm{Z}+\mathrm{R}+\mathrm{H}+\mathrm{G}, \mathrm{HMM}$ & & 103.78 \\
\hline $\mathrm{Z}+\mathrm{R}+\mathrm{H}+\mathrm{G}, \mathrm{MLP}+\mathrm{HMM} ; \mathrm{Z}+\mathrm{R}, \mathrm{HMM}$ & 17.07 & 102.47 \\
\hline $\mathrm{Z}+\mathrm{R}+\mathrm{H}+\mathrm{G}, \mathrm{MLP}+\mathrm{HMM} ; \mathrm{Z}+\mathrm{H}, \mathrm{HMM}$ & 17.96 & 97.19 \\
\hline $\mathrm{Z}+\mathrm{R}+\mathrm{H}+\mathrm{G}, \mathrm{MLP}+\mathrm{HMM} ; \mathrm{Z}+\mathrm{G}, \mathrm{HMM}$ & 15.99 & \\
\hline & & \\
\hline
\end{tabular}

Therefore, the Table 2 showed that having a gain in precision means to a loss of rapidity

\section{Conclusion and Perspectives}

The ultimate objective of this study was to carry out both comparisons which the first one is between the performances in terms of precision and rapidity of four hybrid methods of features extraction which are the zoning combined with Radon then with Hough transforms then with Gabor filter, afterwards combined in the same time with all these descriptors. While the second comparison is achieved between three classifiers that are the multi-layer perceptron, hidden Markov model and a hybrid classifier based on a combination between them. For this purpose we have used in order to pre-process each numeral image the median filter, the thresholding, the centering and the edge detection techniques. We have concluded concerning the features extraction phase that the most precise methos is zoning combined with Radon, Hough transforms and Gabor filter but it is the most slow, while in the framework of classifiers the most precise is that realized via a combination between perceptron multi-layer and hidden Markov model but it is the less fast. 
Moreover, we will hope later on to introduce more other hybrid methods of features extraction and other hybrid classifiers in order to compare between their performances.

\section{References}

[1] R. Salouan, S. Safi and B. Bouikhalene, "Isolated Handwritten Roman Numerals Recognition using Dynamic Programming”, Naïve Bayes and Support Vectors Machines, International Journal of Computer Applications (0975 - 8887), vol. 113, no. 19, (2015) March.

[2] R. Salouan, S. Safi and B. Bouikhalene, "A Comparison between the Self-Organizing Maps and the Support Vectors Machines for Handwritten Latin Numerals Recognition", International Journal of Innovation and Scientific Research, Innovative Space of Scientific Research Journals, ISSN 2351-8014, vol. 7, no. 1, (2014) August 2014, pp. 50-56.

[3] S. V. Rajashekararadhya and V. P. Ranjan, "Efficient zone based feature extraction algorithm for handwritten numeral recognition of four popular south Indian scripts", Journal of Theoretical and Applied Information Technology, (2005), pp. 1171- 1181.

[4] J. Chandaran and M. Kapadia, "Optical Character Recognition", International Journal of Emerging Technology and Advanced Engineering Website: www.ijetae.com ISSN 2250-2459, ISO 9001:2008, Certified Journal, vol. 4, Issue 5, (2014) May.

[5] T. Arodz, "Invariant Objedt Recognition using Radon Transform-Basedfor", Computing and Informatics, vol. 24, (2005), pp. 183-199.

[6] H. Hassen and M. Khemakhem, "A Comparative study of Arabic handwritten characters invariant feature", (IJACSA) International Journal of Advanced Computer Science and Applications, vol. 2, no. 12, (2011).

[7] S. Touj, N. B. Amara and H. Amiri, "Generalized Hough Transform for Arabic Printed Optical Character Recognition", The International Arab Journal of Information Technology, vol. 2, no. 4, (2005) October.

[8] S. Rasheed, A. Naeem and O. Ishaq, "Automated Number Plate Recognition Using Hough Lines and Templates Machones", Proceedings of the World Congress on Engineering and Computer Science, WCECS, vol. I, (2012) October 24-26, San Francisco, USA.

[9] H. Hassen and M. Khemakhem, "A Comparative study of Arabic handwritten characters invariant feature", (IJACSA) International Journal of Advanced Computer Science and Applications, vol. 2, no. 12, (2011).

[10] C. Nusaibath and P. M. A. Mol, "Off-line Handwritten Malayalam Character Recognition Using Gabor Filters", International Journal of Computer Trends and Technology (IJCTT), vol. 4, Issue 8, (2013) August.

[11] S. Singh, A. Aggarwal and R. Dhir, "Use of Gabor Filters for Recognition of Handwritten Gurmukhi Character", International Journal of Advanced Research in Computer Science and Software Engineering, vol. 2, Issue 5, (2012) May.

[12] R. Salouan, S. Safi and B. Bouikhalene, "Printed Arabic Noisy Characters Recognition Using the Multilayer Perceptron", International Journal of Innovation and Scientific Research, Innovative Space of Scientific Research Journals, ISSN 2351-8014, vol. 9, no. 1, (2014) September, pp. 61-69.

[13] A. P. Nagare. "License Plate Character Recognition System using Neural Network", International Journal of Computer Applications, vol. 25, no. 10, (2011) July, pp. 36-39.

[14] C. M. Bishop, "Neural Networks for Pattern Recognition", Department of computer Science and applied mathematics Aston University, Birmingham, UK Clarndon press, (1995).

[15] A. A. D. Gujarati, "Handwritten numeral optical character reorganization through neural network", Pattern Recognition, vol. 43, (2010), pp. 2582-2589.

[16] R. Salouan, S. Safi and B. Bouikhalene, "A Comparative Study between the Hidden Markov Models and the Support Vector Machines for Noisy Printed Numerals Latin Recognition”, International Journal of Innovation and Scientific Research, Innovative Space of Scientific Research Journals, ISSN 2351-8014, vol. 5, no. 1, (2014) July, pp. 16-24.

[17] R. Salouan, S. Safi and B. Bouikhalene, "Printed Eastern Arabic Noisy Numerals Recognition Using Hidden Markov Model and Support Vectors Machine”, International Journal of Innovation and Applied Studies, Innovative Space of Scientific Research Journals, ISSN 2028-9324, vol. 9, no. 3, (2014) November, pp. 1032-1042.

[18] M. Pechwitz and V. Maergner, "HMM Based Approach for Handwritten Arabic Word Recognition Using the IFN/ENIT Database", Proceedings of the Seventh International Conference on Document Analysis and Recognition, IEEE Computer Society, vol. 2, (2003), pp. 890.

[19] S. A. Mahmoud and S. M. Awaida, "Recognition of off-line handwritten Arabic (Indian) numerals using multi-scale features and supports vectors machines vs. hidden Markov models", The Arabian Journal for Science and Engineering, vol. 34, no. 2B, (2009) October.

[20] T. Peter, "The Radon Transform - Theory and Implementation", PhD thesis, Dept. of Mathematical Modelling Section for Digital Signal Processing of Technical University of Denmark, (1996).

[21] C. Høilund, "The Radon Transform", Aalborg University, VGIS, (2007).

[22] P. V. C. Hough, "Method and means for recognizing complex patterns", U.S. patent 3069654, (1962). 
[23] J. Kamarainen, "Feature extraction using Gabor filters", PhD thesis, Lappeenranta University of Technology, (2003).

[24] P. N. Belhumeur, J. P. Hespanha, et al., "Eigenfaces, Eigenfaces vs Fisher faces: «recognition using class specific linear projection”, IEEE Trans. Pattern Analysis and Machine Intelligence, vol. 20, no. 7, (1997), pp. 711- 720.

[25] P. Dymarski, "Hidden Markov models, theory and applications”, InTech Janeza Trdine 9, 51000 Rijeka, Croatia Copyright (C) 2011 InTech.

\begin{abstract}
Authors
R. Salouan, he received his Master's degree in 2010 from Faculty of Sciences and Technology University Sultan Moulay Slimane Beni Mellal Morocco, currently working on his $\mathrm{Ph}$. D in Sultan Moulay Slimane University. His current research interests include pattern recognition, image analysis, document processing and automatic processing of natural languages using hidden Markov models and neural networks.

S. Safi, he was born in Beni Mellal, Morocco in 1971, received the B.Sc. degree in physics (option electronics) from Cadi Ayyad University, Marrakech, Morocco in 1995, M.Sc. and Doctorate degrees from Chouaib Doukkali Univer-sity and Cadi Ayyad University, Morocco, in 1997 and 2002, respectively. He has been a professor of information theory and telecommunication systems at the National School for Applied Sciences, Tangier Morocco, from 2003 to 2005. Since 2006, he is a professor of applied mathematics and programming at the Faculty of Science and Technics, Beni Mellal Morocco. In $2008 \mathrm{He}$ received the Ph.D. degree in telecommunication and informatics from the Cadi Ayyad University. His general interests span the areas of communications and signal processing, estimation, time-series analysis, and system identification - subjects on which he has published 10 journal papers and more than 40 conference papers. Current research topics focus on transmitter and receiver diversity techniques for single- and multi-user fading communication channels, and wide-band wireless communication systems.
\end{abstract}

B. Bouikhalene, he received his Ph.D degree on mathematics in 2001 and Master's degree on Science of Computer and Telecommunications in 2007 from the University Ibn Tofel Kenitra. Currently is a professor in the Sultan Moulay Slimane University Beni Mellal Morocco. His research topics are: the pattern recognition, artificial intelligence and mathematics and its applications. 